\title{
La Collection Islam Burkina Faso
}

\section{Promesses et défis des humanités numériques}

\author{
Frédérick Madore
}

Citer cet article : Frédérick Madore (2021), « La Collection Islam Burkina Faso: promesses et défis des humanités numériques ", Revue d'Histoire Contemporaine de l'Afrique, en ligne. URL :

https://oap.unige.ch/journals/rhca/article/view/stcmadore

Mise en ligne : 15 décembre 2021

DOI : https://doi.org/10.51185/journals/rhca.2021.e610

\section{Résumé}

Cet article propose une réflexion sur les possibilités inédites qu'offre le numérique pour développer de nouvelles méthodes de recherche et de diffusion de données sur l'histoire de l'islam en Afrique de l'Ouest, ainsi que quelques considérations méthodologiques, technologiques et éthiques soulevées par de telles initiatives. Au centre de ces considérations se trouve la Collection Islam Burkina Faso. Ce projet de base de données numérique en libre accès, que j'ai lancé en 2021 et qui est hébergée par les bibliothèques George $A$. Smathers de l'Université de Floride (UF), contient actuellement plus de 2500 documents d'archives, articles de la presse généraliste, publications islamiques sous diverses formes et photographies, en plus de 200 références bibliographiques liées à l'islam et aux musulmans du Burkina Faso (https://islam.domains.uflib.ufl.edu/s/bf-fr). Le texte propose également un bref état des lieux des humanités numériques dans le champ des études africanistes et plus spécifiquement sur l'islam.

Mots-clés : humanités numériques ; archives ; presse ; numérisation ; islam ; Omeka

The Islam Burkina Faso Collection: Promises and Challenges of Digital Humanities

\section{Abstract}

This article reflects on the unprecedented opportunities that digital technology offers for developing new methods of research and data dissemination on the history of Islam in West Africa, as well as some of the methodological, technological, and ethical issues raised by such initiatives. At the center of these considerations is the Islam Burkina Faso Collection. This open access digital database project, which I launched in 2021 and which is hosted by the George A. Smathers Libraries at the University of Florida, currently contains over 2,500 archival materials, newspaper articles, Islamic publications in various forms, and photographs, in addition to 200 bibliographic references related to Islam and Muslims in Burkina Faso (https://islam.domains.uflib.ufl.edu/s/bf/). The paper also provides a brief overview of digital humanities in the field of African studies and, more specifically, on Islam.

Keywords: digital humanities; archives; press; digitalization; islam; Omeka 
Dans cet article, je souhaite réfléchir sur les possibilités inédites qu'offre le numérique pour développer de nouvelles méthodes de recherche et de diffusion de données sur l'histoire de I'islam en Afrique de l'Ouest ainsi que quelques considérations méthodologiques, technologiques et éthiques soulevées par de telles initiatives. Au centre de cette réflexion se trouve la Collection Islam Burkina Faso'. Ce projet de base de données numérique en libre accès, que j'ai lancé en 2021 et qui est hébergée par les bibliothèques George A. Smathers de I'Université de Floride (UF), contient actuellement plus de 2500 documents d'archives, articles de la presse généraliste, publications islamiques sous diverses formes et photographies, en plus de 200 références bibliographiques liées à l'islam et aux musulmans du Burkina Faso (https://islam.domains.uflib.ufl.edu/s/bf-fr). Ce matériel a été obtenu dans le cadre de mes recherches doctorales (2013-2018), portant sur I'histoire du militantisme islamique chez les jeunes et les femmes au Burkina Faso et en Côte d'Ivoire depuis les années 1970, et au cours desquelles j'ai pu constituer un vaste corpus de plus de 10000 documents écrits pour les deux pays ${ }^{2}$. Je reviendrai ici, dans un premier temps, sur l'intérêt de telles sources et notamment la presse généraliste et islamique pour retracer l'histoire des communautés musulmanes d'Afrique de I'Ouest depuis les indépendances. La deuxième partie propose un bref état des lieux des humanités numériques dans le champ des études africanistes et plus spécifiquement sur l'islam dans la région. Enfin, la dernière section présente de manière plus détaillée la base de données, qui a été constituée à partir de la plateforme Omeka³, et les pistes potentielles pour développer le projet à court et moyen terme, particulièrement en établissant une collaboration avec d'autres chercheurs et organismes.

\section{L'histoire de I'islam et des communautés musulmanes d'Afrique de l'Ouest à travers la presse}

En comparaison des dépôts d'archives coloniales généralement considérés comme étant riches en documents et très bien organisés, ceux des États postcoloniaux africains, disposant de moyens extrêmement limités, sont souvent présentés comme très fragmentaires. En effet, la plupart sont caractérisés par la rareté des documents, leur indisponibilité ou même la disparition de fonds entiers, en plus d'être désordonnés car parfois dispersés dans plusieurs centres et ne reposant pas sur une catégorisation efficace: "Archives for the postcolonial period are as scattered, contingent and partial as the history they imperfectly capture ${ }^{4}$. Ainsi, nombre d'historiens de I'Afrique postcoloniale se rabattent sur les sources issues des archives des gouvernements étrangers, des organisations humanitaires et de la presse internationale plutôt que celles des États africains qu'ils étudient ${ }^{5}$. Devant la pauvreté des sources écrites souvent évoquée dans les

\footnotetext{
${ }^{1}$ Le terme « collection » a été privilégié à celui d'« archives », car l'usage du terme archives pour qualifier des projets en humanités numériques est critiqué par de nombreux archivistes, qui préfèrent le terme « collection » ou " collection numérique ». Voir Theimer Kate (2012), "Archives in Context and as Context », Journal of Digital Humanities, 1(2), en ligne. URL: http://journalofdigitalhumanities.org/1-2/archives-in-context-and-as-context-by-kate-theimer/ (consulté le 5 décembre 2021).

2 Madore Frédérick (2018), "Rivalités et collaborations entre aînés et cadets sociaux dans les milieux associatifs islamiques en Côte d'Ivoire et au Burkina Faso (1970-2017) », thèse de doctorat, Québec, Université Laval, en ligne, URL : https://corpus.ulaval.ca/jspui/handle/20.500.11794/33246 (consulté le 5 décembre 2021).

3 https://omeka.org/s (consulté le 5 décembre 2021).

4 White Luise (2015), "Introduction - Suitcases, Roads, and Archives: Writing the History of Africa after 1960 », History in Africa, 42 , p. 266.

${ }^{5}$ Ellis Stephen (2002), «Writing Histories of Contemporary Africa », The Journal of African History, 43(1), pp. 1-26.
} 
recherches en milieu ouest-africain, il n'est dès lors pas étonnant que les enquêtes orales soient considérées comme une source de premier plan y compris par les historiens ${ }^{6}$. Comme l'a souligné Florence Bernault, elle-même historienne, les "historians now wander like born-again ethnographers $»$.

Figure $n^{\circ} 1$ : Archives de presse à la Bibliothèque nationale du Togo à Lomé (photographie de l'auteur)

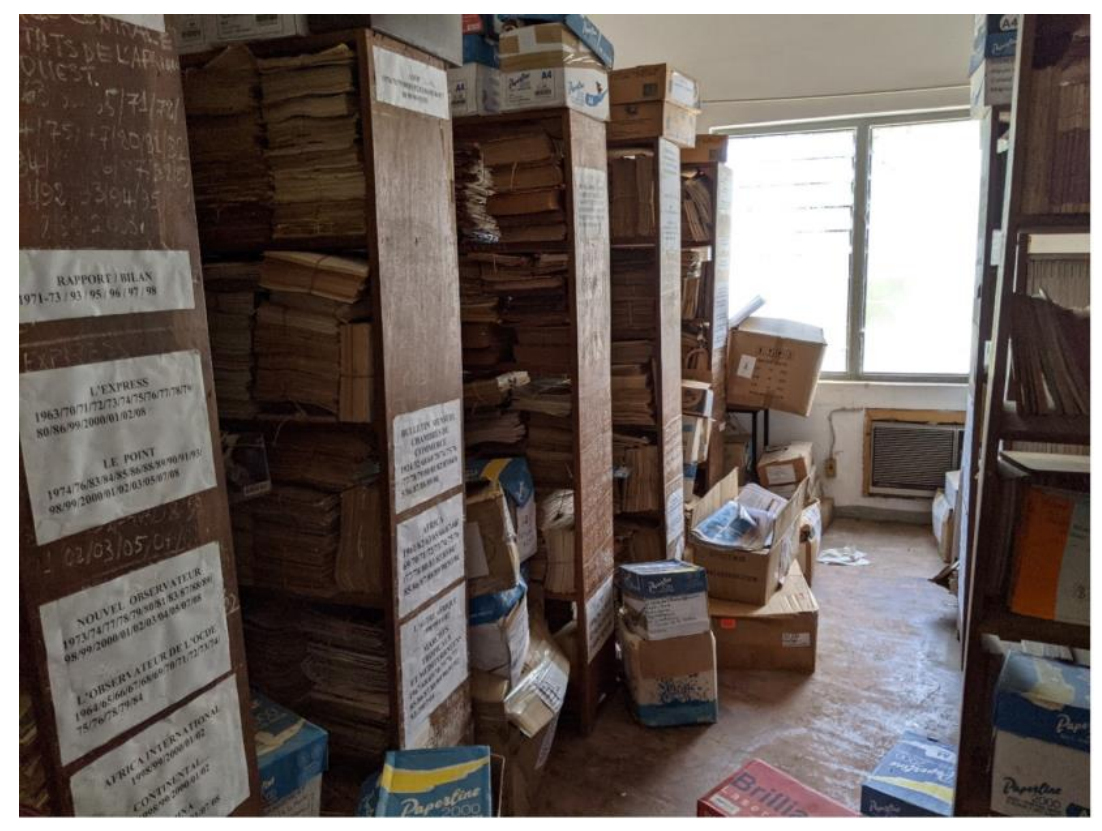

En dépit de nombreuses difficultés, j'ai pu constituer un riche corpus de sources écrites en numérisant plus de 7000 articles de presse portant sur les musulmans au Burkina Faso et en Côte d'Ivoire (célébrations de fêtes religieuses, organisations d'activités islamiques, discours de figures musulmanes, relations avec les pays arabo-musulmans, actions d'ONG islamiques, etc.), ainsi que près de 1000 publications islamiques diverses (brochures, bulletins, magazines, journaux, mensuels, etc.), dont la plupart ont cessé de paraître. Ces documents ont été obtenus à partir des fonds documentaires privés d'individus ${ }^{8}$ et $d^{\prime}$ associations islamiques des deux pays ${ }^{9}$; du Centre de Recherche et d'Actions pour la Paix (CERAP) ${ }^{10}$ et des Archives Nationales de Côte d'Ivoire à Abidjan; de I'Institut de Recherche pour le Développement (IRD) ${ }^{11}$ et du Centre National des

\footnotetext{
${ }^{6}$ White Luise, Miescher Stephan et Cohen David William (dir.) (2001), African Words, African Voices: Critical Practices in Oral History, Bloomington, Indiana University Press.

7 Bernault Florence (2015), "Suitcases and the Poetics of Oddities: Writing History from Disorderly Archives », History in Africa, 42, p. 270.

8 Je suis tout particulièrement redevable à Ibrahim Doumbia, un ancien responsable de l'Association des Élèves et Étudiants Musulmans de Côte d'Ivoire (Aeemci) et journaliste, qui m'a généreusement ouvert ses vastes archives personnelles contenant d'innombrables publications islamiques, dont Plume Libre et Islam Info.

${ }^{9}$ Aeemci à Abidjan; Association des Élèves et Étudiants Musulmans du Burkina (Aeemb) et Cercle d'Études, de Recherches et de Formation Islamiques (Cerfi) à Ouagadougou.

10 https://cerap-inades.org (consulté le 5 décembre 2021).

11 https://www.ird.fr/burkina-faso (consulté le 5 décembre 2021).
} 
Archives à Ouagadougou; du Library of Congress ${ }^{12}$ à Washington D.C. ; et enfin, de l'agrégateur de journaux africains AllAfrica.com ${ }^{13}$.

La constitution d'un tel corpus de sources écrites offre plusieurs avantages. La presse généraliste et les différentes publications islamiques sur support papier sont très riches et constituent un excellent moyen pour retracer avec précision l'histoire des communautés musulmanes. Ceci est particulièrement le cas pour les premières décennies de l'indépendance alors que nombre d'acteurs importants de l'époque sont décédés et que les informations obtenues par le biais des enquêtes orales peuvent comporter des imprécisions. De plus, ces sources écrites offrent à la fois un regard interne et externe sur les activités de différents individus et groupes musulmans. Dans les années 1970 et 1980, la presse généraliste burkinabè ${ }^{14}$ et ivoirienne ${ }^{15}$ offrait une couverture relativement limitée de l'actualité touchant les musulmans. Cela se réduisait généralement à des comptes rendus à l'occasion de fêtes islamiques (Aïd al-Adha, Aïd el-Fitr), lors du pèlerinage à La Mecque, l'inauguration de nouvelles mosquées ou la mort de dignitaires religieux. Cependant, à partir de la fin des années 1980, le volume d'articles consacrés à l'islam a considérablement augmenté avec la couverture assez détaillée de nombreuses activités de différentes associations islamiques, ainsi que des conflits et dissensions internes au sein de la communauté musulmane - prenant souvent une tournure médiatique importante dans les deux pays. Les différents articles comportent aussi des témoignages et interviews de leaders musulmans ou de fidèles « ordinaires ».

Figure $n^{\circ} 2$ : Exemple d'un article de journal (L'Observateur, 23 septembre 1981)

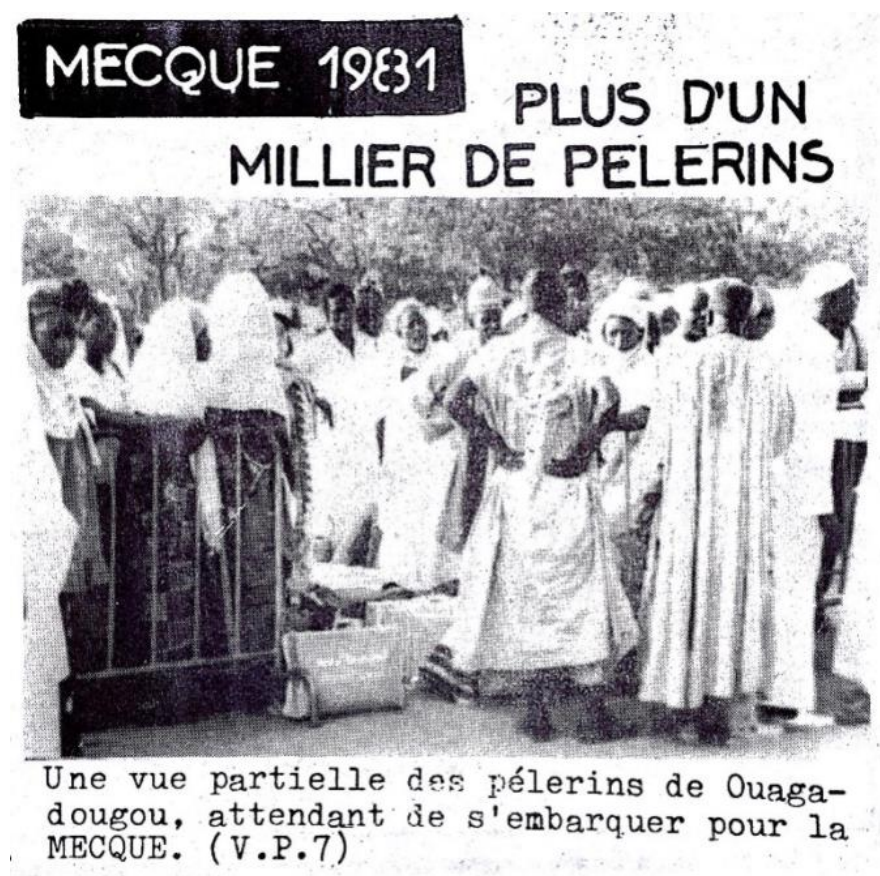

\footnotetext{
12 https://www.loc.gov (consulté le 5 décembre 2021).

13 Cette plateforme, propriété de la société privée AllAfrica Global Media qui opère depuis Le Cap, Dakar, Abuja, Monrovia, Nairobi et Washington D.C., relaie les nouvelles de plus de 140 organismes de presse africains. https://fr.allafrica.com/misc/info/about (consulté le 5 décembre 2021).

14 Carrefour africain, L'Observateur, Sidwaya.

15 Fraternité Matin, Fraternité Hebdo.
} 
Par ailleurs, dans le cadre d'une recherche de terrain sur un sujet sensible dans une région où les défis sont nombreux ${ }^{16}$, surtout depuis la flambée d'attaques djihadistes, il est parfois difficile d'obtenir la confiance de groupes ou d'individus dont les croyances sont a priori considérées comme une menace à la sécurité. Ceci est tout particulièrement le cas pour les mouvements salafis ${ }^{17}$, qui sont abusivement associés à la radicalisation et la violence. Les chercheurs peuvent être pris pour des « espions » des services de renseignements nationaux ou occidentaux dans la lutte antiterroriste ${ }^{18}$. À cela s'ajoute le fait que beaucoup d'acteurs religieux sont tout à fait conscients de ce qui s'écrit sur eux. Cette méfiance s'était d'ailleurs manifestée dans le cadre de mes recherches avec les groupes salafis ivoiriens, dont les leaders, très préoccupés à l'idée que leur mouvement intéresse des chercheurs étrangers, avaient affirmé ne pas vouloir participer à des projets de recherche. Or, ce problème d'accès fut largement comblé par la presse islamique et généraliste, qui m'ont permis d'analyser le développement rapide du salafisme en Côte d'Ivoire depuis les années $1990^{19}$.

De plus, lors d'un entretien avec un chercheur occidental, des figures religieuses importantes ou des musulmans lambda peuvent tenir des discours très policés alors qu'ils peuvent avoir une prise de parole un peu plus libérée s'ils sont interrogés dans le cadre d'une publication islamique, qui n'est pas destinée a priorià un public non musulman. À cela s'ajoute le fait qu'il est plus difficile de mener des entretiens auprès de femmes appartenant à des milieux salafis en étant un homme. Ainsi, les interviews de musulmanes et les comptes-rendus d'activités islamiques strictement féminines dans la presse favorisent le croisement d'informations obtenues par le biais d'enquêtes orales. Ils permettent aussi d'atténuer l'avantage que les chercheures étrangères peuvent détenir sur leurs collègues masculins en appartenant à un " troisième genre $^{20} »$, qui facilite l'accès à la fois aux hommes et aux femmes selon les contextes.

Les sources écrites ont également représenté un formidable outil pour accéder aux discours et prises de position sur diverses questions religieuses, sociales et parfois politiques, tant récents qu'anciens, de différents acteurs musulmans. Outre les débats entourant la laïcité, I'organisation du pèlerinage, l'enseignement confessionnel islamique ou encore les réformes du code de la famille, on peut aussi souligner l'importante médiatisation d'imams ivoiriens, qui se sont prononcés publiquement sur l'«ivoirité » et les traitements infligés aux musulmans dans les

\footnotetext{
${ }^{16}$ Marzo Pietro et Gomez-Perez Muriel (2020), « Faire du terrain au Moyen-Orient et en Afrique : stratégies d'approche et défis de positionnement du chercheur », Recherches qualitatives, 39(1), pp. 1-20.

17 Le salafisme est un mouvement de réforme musulman qui est né vers la fin du XIXe siècle en Égypte, bien que son origine exacte fasse toujours l'objet de débats. Souvent apparenté au wahhabisme qui s'est développé dans l'actuelle Arabie Saoudite au XVIII' siècle sous l'impulsion d'lbn 'Abd al-Wahhab, le salafisme a influencé de nombreux penseurs et servi de base à divers mouvements en islam. II prône une pureté rituelle basée sur le modèle des « pieux prédécesseurs » (salaf al-șālị̂), c'est-à-dire les compagnons du prophète Muhammad, et l'unicité de Dieu (tawhid). Ainsi, les salafis rejettent avec ferveur les confréries soufies (tañqa) et l'incorporation de traditions culturelles locales (divination, utilisation de charmes ou de talismans, vénérations des cheikhs), qui sont assimilées à des innovations illicites (bid'ah) et à de l'associationnisme (shirk). Ils estiment que ces pratiques ont corrompu l'islam et visent à rétablir ce qu'ils considèrent comme l'islam véritable et authentique pratiqué par les trois premières générations de musulmans. Voir Østebø Terje (2015), "African Salafism: Religious Purity and the Politicization of Purity », Islamic Africa, 6(1-2), pp. 1-29.

18 Prud'homme Pierre (2015), "L'imam, l'expert et le diplomate : retour sur une recherche au Haut Conseil Islamique du Mali », Civilisations, 64(1), pp. 129-140.

${ }^{19}$ Madore Frédérick (2016), «The New Vitality of Salafism in Côte d'Ivoire: Toward a Radicalization of Ivoirian Islam? », Journal of Religion in Africa, 46(4), pp. 417-452.

20 Schwedler Jilian (2006), "The Third Gender: Western Female Researchers in the Middle East ", Political Science \& Politics, 39, pp. 425-428.
} 
années 1990 et 200021. Ceci m'a permis d'adopter une approche résolument empirique en donnant un large accès aux discours des acteurs étudiés.

Figure $n^{\circ} 3$ : Première page du journal Le Jour (9 août 1995)
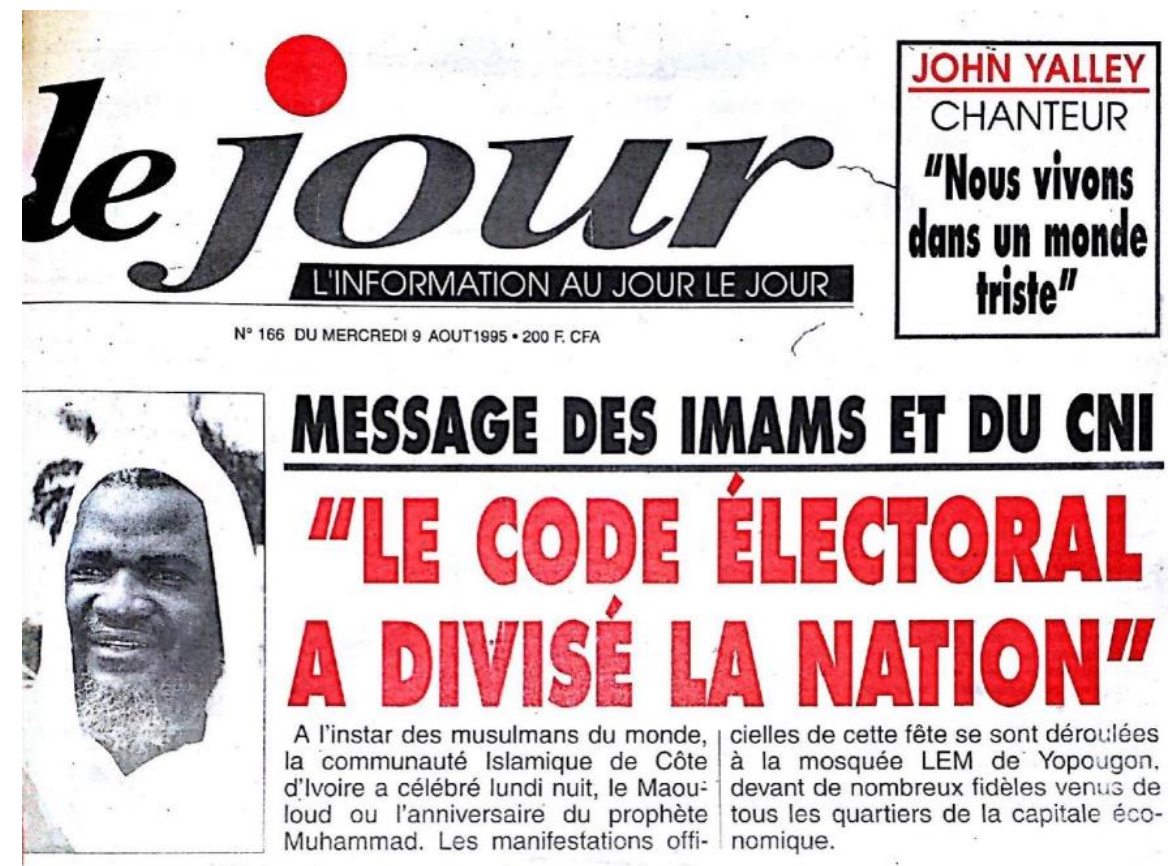

Enfin, la presse généraliste est très révélatrice de l'influence médiatique dont bénéficient différentes figures et associations islamiques. Par exemple, au Burkina Faso, de jeunes «intellectuels musulmans» francophones ont éclipsé les porte-paroles traditionnels de leur communauté durant le processus de transition suivant la chute du président Blaise Compaoré en octobre 201422. En effet, la presse s'avère très utile pour analyser les rapports de pouvoir qui soustendent l'accès à la sphère publique et la participation de groupes qui sont habituellement exclus des débats publics tels que les jeunes et les femmes. La comparaison entre les journaux proches du pouvoir et la presse de l'opposition permet de voir comment les médias progouvernementaux accordent un traitement privilégié et donnent une large visibilité à certains groupes musulmans plus proches de l'État au détriment d'autres mouvements et dirigeants soupçonnés d'être proches de l'opposition ${ }^{23}$.

\footnotetext{
${ }^{21}$ Par exemple, la déclaration du Conseil supérieur des imams appelant à « réexaminer, dès que possible, les aspects contestés du [nouveau] code électoral », qui excluait la candidature d'Alassane Ouattara à l'élection présidentielle d'octobre 1995, se trouvait à la une des journaux Le Jour et La Voie. Voir "Message des imams et du CNI : "Le Code électoral a divisé la nation" ", Le Jour, 9 août 1995 ; «Pour prévenir tout risque d'éclatement social : les imams appellent à un réexamen du code électoral », La Voie, 9 août 1995. 22 Madore Frédérick (2020), "Francophone Muslim Intellectuals, Islamic Associational Life and Religious Authority in Burkina Faso », Africa, 90(3), pp. 625-646.

${ }^{23} \mathrm{Ce}$ fut tout particulièrement le cas de la couverture, par la presse ivoirienne, des différentes associations islamiques habilitées à organiser le pèlerinage à La Mecque dans les années 1990 et 2000. Ainsi, durant la présidence d'Henri Konan Bédié, l'organisation des différents pèlerinages par le Conseil supérieur islamique, qui était réputé être à la solde du pouvoir, bénéficia d'une couverture médiatique exagérée et démesurément positive. Voir Madore Frédérick et Traoré Yssoufou (2018), "L'organisation du hadj en Côte d'Ivoire : entre facteur de cohésion et source de rivalités au sein de la communauté musulmane (1993-2010) ", Cahiers d'études africaines, 229, pp. 179-208.
} 


\section{Humanités numériques et études africanistes : des progrès en demi- teinte}

À l'instar d'autres champs d'études, la facilité avec laquelle les chercheur·e.s africanistes peuvent dorénavant accéder à un large éventail de ressources en ligne, gratuitement ou de manière payante, ont transformé la nature du processus de recherche et ont offert de nouvelles opportunités. En effet, les programmes de numérisation d'archives africaines, publiques et privées, se sont multipliés depuis les années $2000^{24}$. On peut notamment évoquer l'African Online Digital Library $(\mathrm{AODL})^{25}$, le SlaveVoyages (Trans-Atlantic and Intra-American slave trade databases) ${ }^{26}$, le Endangered Archives Programme de la British Library ${ }^{27}$, ou encore l'impressionnant travail effectué par Derek R. Peterson de l'Université du Michigan sur I'Ouganda ${ }^{28}$. Les archives d'un nombre croissant de journaux africains sont désormais disponibles en ligne, bien que cela concerne davantage la presse anglophone que francophone ${ }^{29}$. Outre des projets financés par des institutions américaines et britanniques, des initiatives africaines venant de plusieurs bibliothèques, centres d'archives et universités du continent ont été lancées avec succès. L'Afrique du Sud et le Nigeria ont été particulièrement dynamiques à cet égard ${ }^{30}$. Bien que le continent demeure encore quelque peu en marge du développement des humanités numériques, un réseau pour les humanités numériques en Afrique ${ }^{31}$ s'est constitué en 2019 à la suite du colloque annuel de I'Alliance of Digital Humanities Organizations (ADHO). D'autres conférences ont aussi été organisées sur ce sujet, dont la quatrième édition du Princeton African Humanities Colloquium en 2020, qui avait pour thème "Africa and Digital Humanities ${ }^{22}$ ». À la lumière de ces développements, un nombre croissant d'auteurs ont souligné l'essor du tournant numérique (« digital turn») dans les études africanistes ${ }^{33}$.

Cependant, en dépit de la popularité croissante des humanités numériques, un nombre très limité d'initiatives en lien avec l'islam en Afrique de l'Ouest a tenté de mobiliser les outils du numérique pour traiter et analyser les données, mais surtout pour faciliter la diffusion et le partage des savoirs. Jusqu'à tout récemment, les premières ressources en ligne telles que le portail Islam and Modernity: Alternatives in Contemporary Senegambia \& Ghana ${ }^{34}$ étaient restées au stade de " projet pilote ${ }^{35}$ ». Bien qu'étant indirectement liés à l'islam, le Tombouctou Manuscripts Project ${ }^{36}$, mené par l'Université de Cape Town, et l'African Ajami Library ${ }^{37}$, créé et dirigé par Fallou Ngom,

\footnotetext{
${ }^{24}$ Fouéré Marie-Aude, Rillon Ophélie et Pommerolle Marie-Emmanuelle (2020), « Pourquoi Sources? Rigueur empirique, réflexivité et archivage en sciences humaines et sociales et dans les études africaines », Sources, 1, pp. 9-10.

25 https://aodl.org (consulté le 5 décembre 2021).

26 https://www.slavevoyages.org (consulté le 5 décembre 2021).

${ }^{27}$ https://eap.bl.uk (consulté le 5 décembre 2021).

${ }^{28}$ https://derekrpeterson.com/archive-work (consulté le 5 décembre 2021).

29 Voir par exemple la collection East African Newspapers du Global Press Archive CRL Alliance, qui contient plus de 450000 pages de journaux des années 1940 au début des années 2000. https://www.crl.edu/news/global-press-archive-crl-alliance-makes-secondcopyright-collection-available (consulté le 5 décembre 2021).

30 Voir par exemple le South African Centre for Digital Language Resources (SADiLaR).

31 https://dhafrica.blog (consulté le 5 décembre 2021).

32 https://pah.princeton.edu/colloquia/princeton-2020 (consulté le 5 décembre 2021).

33 Chamelot Fabienne, Hiribarren Vincent et Rodet Marie (2020), "Archives, the Digital Turn, and Governance in Africa », History in Africa, 47, pp. 101-118.

${ }^{34}$ Foley Catherine (2014), «Developing Materials for a Digital Library Gallery », Islamic Africa, 5(1), pp. 83-90 ; Robinson David (2014),

«Muslim Societies in West Africa: Historical and Contemporary Perspectives in Digital Form », Islamic Africa, 5(1), pp. 103-121.

35 Limb Peter (2014), «Islamic Africa: A Select, Annotated Webography », Islamic Africa, 5(1), pp. 91-102.

${ }^{36} \mathrm{http}: / /$ www.tombouctoumanuscripts.uct.ac.za (consulté le 5 décembre 2021).

37 https://open.bu.edu/handle/2144/1896 (consulté le 5 décembre 2021).
} 
sont particulièrement dignes de mention. Plus récemment, Zachary Wright a lancé le Tijani Literature Project ${ }^{38}$, une base de données numérique de manuscrits en arabe à propos de la Tijaniyya, l'ordre soufi le plus important d'Afrique.

Bien que les perspectives et les promesses offertes par les technologies numériques soient nombreuses pour les études africanistes, nombre de chercheurs et d'archivistes ont toutefois souligné le problème de la « fracture numérique » (" digital divide») en raison de l'accessibilité $d^{\prime}$ Internet qui demeure encore limitée en Afrique subsaharienne ${ }^{39}$. D'autres ont aussi soulevé les problèmes liés à la propriété intellectuelle des sources numériques ou encore le bouleversement des pratiques et normes archivistiques. La visite de chercheurs étrangers dans les centres d'archives africains peut également constituer une source de revenus non négligeable pour ces établissements. À cela s'ajoutent les enjeux politiques de l'archivage numérique, qui tendent à reproduire les hiérarchies coloniales alors que certains pays ou institutions sont privilégiées pour les possibilités de financement. En effet, la plupart des projets de numérisation, qui sont souvent coûteux, sont financés par des institutions du Nord ou sont menés par des sociétés privées dont l'objectif est le profit financier plutôt que la large diffusion gratuite des connaissances ${ }^{40}$. Ainsi, certains ont dénoncé l'« impérialisme numérique ${ }^{41}$ » et le "Digital Savior Complex ${ }^{42}$ » comme ce fut le cas de Peter Limb, historien spécialiste de l'Afrique du Sud, qui, dès 2007, avait affirmé que "Twenty-first century missionaries carry not Bibles but scanners ${ }^{43}$ ". L'archivage en ligne des données de recherche, consultables sans barrière financière, constitue néanmoins une alternative intéressante pour les chercheurs tant ouest-africains qu'étrangers pour pallier les difficultés souvent rencontrées dans les bibliothèques et les centres d'archives de la région ${ }^{44}$.

\section{Une base de données numérique avec Omeka : un projet en constante évolution}

L'idée de diffuser et d'archiver en accès ouvert (" open access») une large partie de mon corpus de sources, afin que ces documents soient consultables par des chercheurs du monde entier pour mener de nouvelles études sur l'islam au Burkina Faso, a donc été fortement influencée par les humanités numériques ainsi que les réflexions entourant la «science ouverte ${ }^{45} »$. J'ai été particulièrement inspiré par la démarche d'Enrique Martino, qui a créé un site Internet sur lequel se trouve la quasi-totalité des sources d'archives qu'il a utilisée pour sa thèse sur l'histoire de la Guinée Équatoriale ${ }^{46}$. Ainsi, grâce à un financement du programme de bourses

\footnotetext{
38 https://tijani.org (consulté le 5 décembre 2021).

39 Barringer Terry et Wallace Marion (dir.) (2014), African Studies in the Digital Age: Dis/Connects?, Leiden, Brill.

${ }^{40}$ Chamelot F., Hiribarren V. et Rodet M., " Archives, the Digital Turn », art. cité ; Fouéré M.-A., Rillon O. et Pommerolle M., " Pourquoi Sources? », art. cité, p. 10.

${ }^{41}$ Breckenridge Keith (2014), "The Politics of the Parallel Archive: Digital Imperialism and the Future of Record-Keeping in the Age of Digital Reproduction », Journal of Southern African Studies, 40(3), pp. 499-519.

${ }^{42}$ Shringarpure Bhakti (2020), " Africa and the Digital Savior Complex », Journal of African Cultural Studies, 32(2), pp. 178-194.

43 Limb Peter (2007), "The Politics of Digital "Reform and Revolution": Towards Mainstreaming and African Control of African Digitisation ", Innovation, 34, p. 23.

44 Daly Samuel Fury Childs (2017), « Archival Research in Africa », African Affairs, 116(463), pp. 311-320.

${ }^{45}$ La science ouverte, qui se veut au service du bien commun, promeut notamment le libre partage des connaissances scientifiques et des données de recherche et bibliographique sans barrière financière afin d'instaurer une meilleure justice cognitive dans les pays des Suds. Voir Piron Florence, Régulus Samuel et Dibounje Madiba Marie Sophie (dir.) (2016), Justice cognitive, libre accès et savoirs locaux : pour une science ouverte, juste, au service du développement local durable, Québec, Éditions science et bien commun.

${ }^{46}$ Martino Enrique (2014), "Open Sourcing the Colonial Archive - A Digital Montage of the History of Fernando Pó and the Bight of Biafra ", History in Africa, 41, pp. 387-415. L'auteur a également consacré un billet de blogue sur les questions éthiques et juridico-
} 
postdoctorales Banting du Canada et au soutien inestimable de Perry Collins et de Tiffany Esteban des Bibliothèques George A. Smathers de I'UF ainsi que de Leonardo A. Villalón du Sahel Research Group, j'ai pu mettre en ligne une base de données numérique novatrice, et en accès libre, de documents de différentes formes liés à l'islam au Burkina Faso. Elle a été constituée avec la plateforme Omeka, qui est développée par le Center for History and New Media (CHNM) de I'Université George Mason ${ }^{47}$. Ce logiciel gratuit et open source, qui est notamment utilisé par de nombreux musées, bibliothèques, sociétés historiques, centre d'archives et chercheur·e.s, permet la publication sur le web de fichiers de tout type (vidéo, audio, image, texte) et leur identification par l'attribution de métadonnées afin de faciliter la conservation et la consultation de collections numériques. Omeka permet également de générer un site web facile à administrer et dit "réactif», dont la consultation est adaptée pour des formats d'écran différents (ordinateur, tablette, téléphone portable), et accessible avec des connexions Internet limitées.

En plus de l'attribution de métadonnées selon le format Dublin Core ${ }^{48}$ relatif au contenu (titre, sujet, description, langue), à la propriété intellectuelle (créateur, éditeur, source, gestion de droits d'auteur) et à l'instanciation (date, type), la reconnaissance optique de caractères (ROC) a aussi été appliquée à chaque document afin d'indexer le texte intégral. De cette manière, il est donc possible d'effectuer une recherche simple par mots-clés, ou avancée en croisant plusieurs critères parmi les milliers de documents de la base de données. Ceci permet également un référencement efficace pour améliorer le positionnement des pages du site web dans les résultats des moteurs de recherche. Un index des métadonnées « sujet » a aussi été produit, qui répertorie plus de 600 mots-clés identifiant les différents thèmes du contenu du document tels que : les individus et les associations musulmanes impliqués (« imam X», «AEEMB (Association des Élèves et Étudiants Musulmans du Burkina)») ; les lieux (« Grande mosquée de Zangouettin », «Institut islamique El Nour ») ; les sujets dont il est question ("laïcité », " voile ») et certains évènements importants de l'actualité burkinabè ("Projet de loi sur les libertés religieuses (2016-2017)»). Le site, dont l'interface est bilingue (français-anglais), offre aussi la possibilité de télécharger la version PDF des versions numérisées des documents.

Figure $n^{\circ} 4$ : Exemple d'un document avec métadonnées et texte intégral obtenu avec la ROC

\footnotetext{
politiques liés à la gestion des droits d'auteur pour la diffusion en ligne de documents provenant de bibliothèques et fonds d'archives de différents pays européens et africains: "Law, License and Digital Reproduction in the Colonial Archive ", 17 février 2014, http://www.opensourceguinea.org/2014/02/law-license-and-digital-reproduction-in.html (consulté le 5 décembre 2021).

${ }^{47}$ Le CHNM est aussi à l'origine du logiciel de gestion de références bibliographiques Zotero.

${ }^{48}$ Dublin Core est une norme internationalement reconnue pour la description de toute ressource.
} 


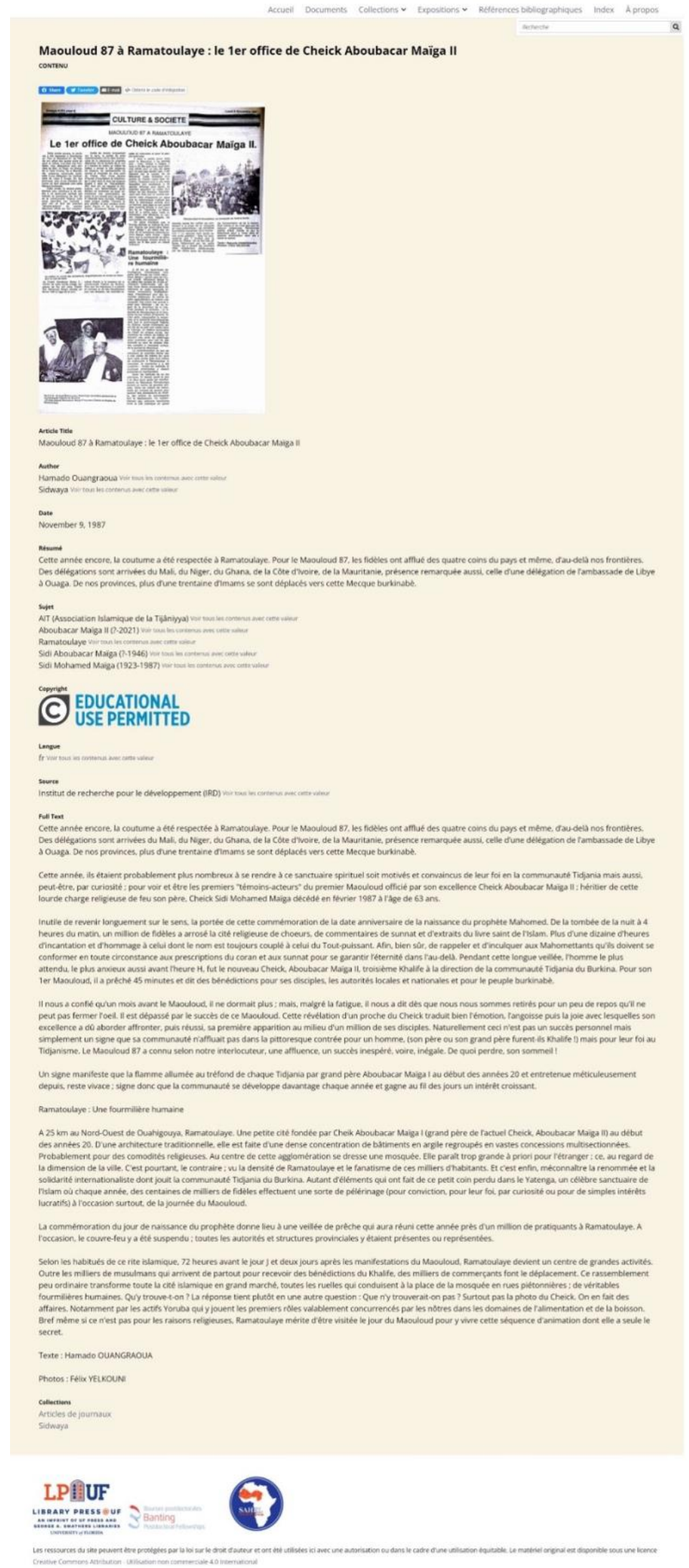

Tous les documents sont regroupés par « collections » thématiques selon leur type : articles de la presse généraliste (Carrefour africain [1962-1987], L'Observateur [1979-1984], Sidwaya [19832019], L'Observateur Paalga [1991-2019], et Le Pays [1992-2019]), journaux islamiques (A/ 
Mawadda49, An-Nasr Trimestriep0, An-Nasr Vendred ${ }^{11}$ et La Preuve52) et photographies. Enfin, le site propose plus de 200 références d'ouvrages, chapitres de livre, articles de revue, mémoires et thèses - incluant celles soutenues dans des universités africaines, qui sont souvent mal référencées - en lien avec I'islam au Burkina Faso. Ces références sont facilement importables avec les logiciels de gestion bibliographique comme Zotero.

Figure $n^{\circ} 5$ : Collections thématiques

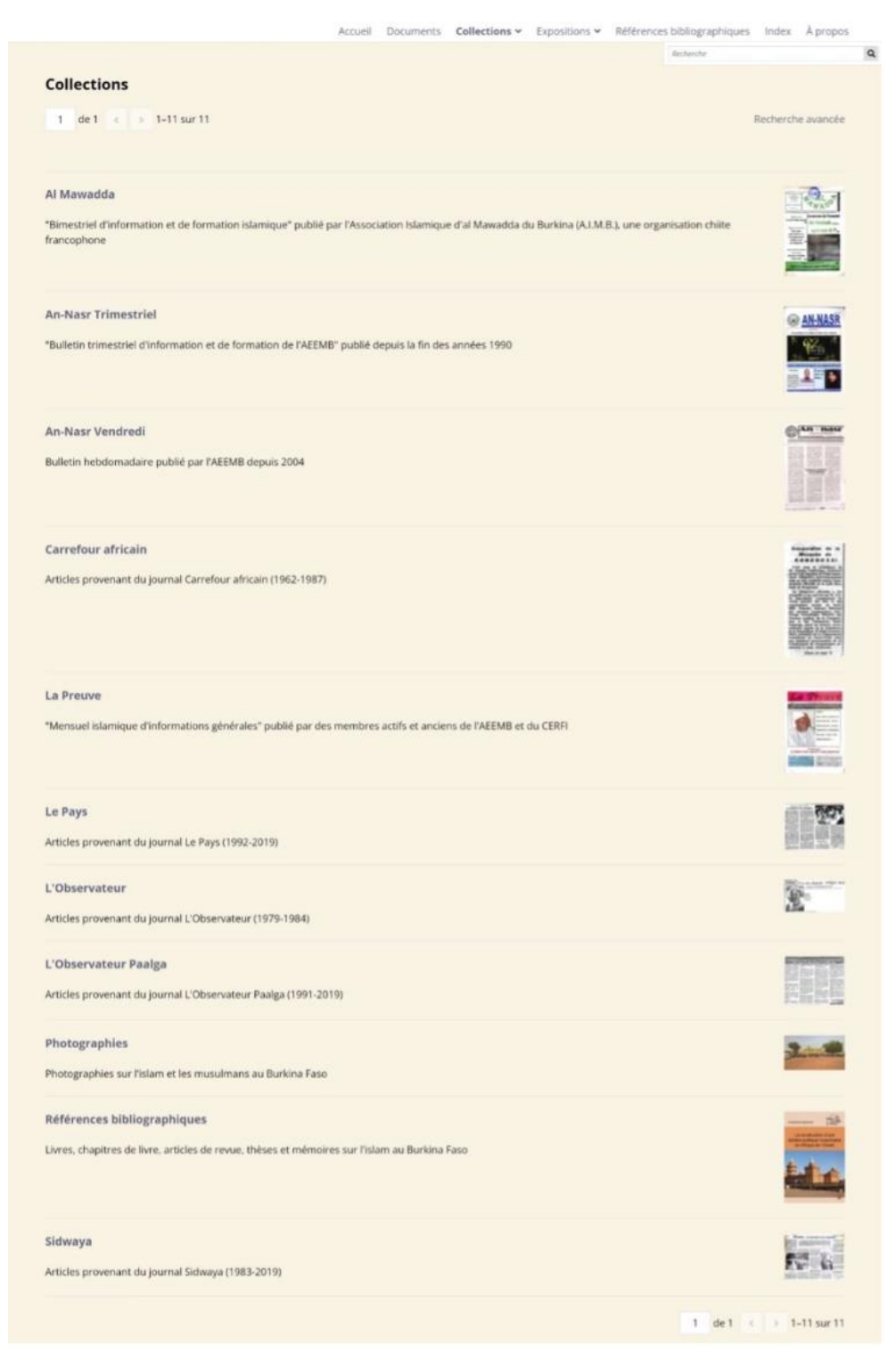

Un des défis majeurs de tout projet de numérisation et d'archivage de journaux est d'obtenir le consentement du titulaire des droits d'auteur, ce qui est souvent difficile. Ceci est d'autant plus vrai que les historiens sont généralement peu formés sur ces questions juridiques.

\footnotetext{
49 «Bimestriel d'information et de formation islamique » publié par l'Association Islamique d'al Mawadda du Burkina (AIMB), qui a commencé à paraître vers 2000-2001.

50 « Bulletin trimestriel d'information et de formation de l'AEEMB » publié depuis la fin des années 1990

51 Bulletin hebdomadaire publié par l'Aeemb depuis 2004.

52 « Mensuel islamique d'informations générales », publié entre 2007 et 2011.
} 
J'ai donc contacté les éditeurs des journaux Sidwaya, L'Observateur Paalga et Le Pays afin de leur présenter le projet et sa valeur scientifique. Après consultation avec leur service juridique respectif, ils ont gracieusement accepté, en tant que titulaires des droits d'auteur, de signer un " contrat de distribution numérique » m'autorisant à numériser, distribuer et archiver les articles identifiés à des fins éducatives et à but non lucratif via Internet pour le compte de l'Université de Floride. Pour ce qui est des œuvres orphelines (" orphan works»), c'est-à-dire les documents dont le(s) titulaire(s) des droits d'auteur sont impossibles à localiser ou à identifier - et qui se trouvent en quelque sorte dans un vide juridique -, il est possible d'invoquer le "fair use " (usage raisonnable ${ }^{53}$. Dans ce cadre, le matériel peut donc être mis à disposition à des fins éducatives et non lucratives d'autant plus que la transformation d'articles de presse ou d'un journal qui a cessé de paraître en une source primaire historique n'affecte pas la valeur marchande du document original, qui provenait de sa date de vente initiale remontant à plusieurs années ou décennies ${ }^{54}$. C'est donc ce qui a été employé pour les journaux islamiques $A /$ Mawadda et $L a$ Preuve pour lesquels la mention «protégé par le droit d'auteur - titulaire(s) des droits impossible(s) à localiser ou à identifier » a été attribuée. Si un avertissement du détenteur des droits d'auteur ("takedown notice») advenait, les articles ou les journaux seraient alors immédiatement retirés de la base de données et un refus pourrait conduire à des procédures judiciaires ${ }^{55}$.

Dans une prochaine phase du projet, je souhaite, d'une part, l'élargir à la Côte d'Ivoire en y incorporant le matériel que j'ai déjà numérisé par le biais d'un partenariat avec Issouf Binaté, enseignant-chercheur à l'Université Alassane Ouattara de Bouaké. D'autre part, je vais chercher à obtenir l'autorisation d'autres périodiques burkinabè (" contrat de distribution numérique » ou en utilisant le fair use le cas échéant) afin de pouvoir ajouter de nouveaux documents à la base de données existante. Surtout, une collaboration avec les Archives nationales du Burkina Faso à Ouagadougou permettrait d'intégrer les centaines de documents contenus dans le fonds " 7V485 Correspondance des communautés musulmanes au Chef de l'État. Textes réglementaires. 1956 1995 ». La publication des inventaires d'archives, tel que le site web sur les Archives coloniales de I'Afrique Équatoriale Française ${ }^{56}$, pourrait également constituer une option intéressante.

Dans de tels projets, les questions liées à la durabilité numérique et aux stratégies de développement à long terme sont également primordiales. Collaborer avec une bibliothèque universitaire représente un avantage à cet égard. Ainsi, la LibraryPress@UF, qui est une imprimerie de I'University of Florida Press et des bibliothèques George A. Smathers de I'UF, assurera la préservation de la base de données et du site web en l'archivant dans le dépôt institutionnel de

\footnotetext{
53 Enrique Martino s'est d'ailleurs appuyé essentiellement sur le fair use pour son site opensourceguinea.org (consulté le 5 décembre 2021).

54 Hansen David (2016), "Digitizing Orphan Works: Legal Strategies to Reduce Risks for Open Access to Copyrighted Orphan Works », in K. K. Courtney et P. Suber (dir.), Harvard Library, https://dash.harvard.edu/handle/1/27840430 (consulté le 5 décembre 2021).

55 Clause de non-responsabilité : il est à noter que ceci ne représente en aucun cas un conseil juridique professionnel. Veuillez consulter des spécialistes de votre institution pour vous conseiller pour tout projet de numérisation que vous souhaiteriez mettre en œuvre.

56 https://archivescolonialesbrazzaville.wordpress.com (consulté le 5 décembre 2021). Voir aussi https://archivescolonialesabidjan.wordpress.com sur les archives coloniales conservées aux Archives nationales de Côte d'Ivoire (consulté le 5 décembre 2021).
} 
I'institut pendant au moins dix ans. Ceci permettra d'assurer un développement à moyen et long terme du projet. Outre l'amélioration du design graphique du site web, je travaille actuellement à l'ajout d'« expositions numériques » comme points d'entrée pour la collection. Chacune d'entre elles regroupera une sélection de documents de la base de données par thèmes, tels que les musulmans et la laïcité, l'enseignement confessionnel islamique et l'activisme étudiant. En plus de fournir de l'information contextuelle pour approcher ce matériel, une bibliographie sélective viendra compléter la présentation de l'exposition. Les documents pourront aussi être intégrés à une ligne du temps interactive et être géolocalisés afin de pouvoir les parcourir sur une carte.

À moyen et long terme, j'ai l'intention d'utiliser ce projet comme une vitrine pour obtenir un financement afin de construire une base de données numérique collaborative plus large sur I'islam en Afrique de l'Ouest, qui inclurait non seulement du matériel provenant de mes nouvelles recherches sur le Bénin et le Togo, mais également d'autres pays et chercheurs. En effet, l'interface d'Omeka offre un ensemble de fonctions administratives et de permissions d'utilisateur, qui permettent d'accorder un accès différencié selon le type "chercheur », "éditeur » ou "administrateur». Ainsi, selon un modèle de recherche collaborative, d'autres spécialistes pourraient participer au projet en créant leurs propres collections sur des thèmes particuliers ou en alimentant la base de données de leurs propres sources dans le respect des droits d'auteur selon la démarche telle qu'énoncée précédemment ${ }^{57}$. Ceci m'apparaît une piste d'autant plus intéressante que la plupart des chercheurs de nos jours stockent des gigaoctets de photographies d'archives avec leur téléphone portable.

Par ce projet, j'espère pouvoir démocratiser l'accès à des documents de diverses natures sur l'islam au Burkina Faso, paver la voie à de nouvelles recherches sur ce sujet et surtout servir de modèle pour d'autres initiatives similaires. Les humanités numériques ont la capacité de développer la recherche en histoire sur l'Afrique en plus de valoriser l'interdisciplinarité et la collaboration entre chercheurs de différentes régions du monde pour la production, le partage, le traitement et la publication des connaissances.

Frédérick Madore

Leibniz-Zentrum Moderner Orient (ZMO) (Allemagne)

\section{Bibliographie}

BARRINGer Terry et Wallace Marion (dir.) (2014), African Studies in the Digital Age: Dis/Connects?, Leiden, Brill.

Bernault Florence (2015), "Suitcases and the Poetics of Oddities: Writing History from Disorderly Archives », History in Africa, 42, pp. 269-277.

\footnotetext{
57 Louis Audet Gosselin a d'ailleurs accepté de partager diverses publications islamiques du Burkina Faso de sa collection personnelle, dont plusieurs documents se trouvent déjà en ligne.
} 
BRECKENRIDGE Keith (2014), "The Politics of the Parallel Archive: Digital Imperialism and the Future of Record-Keeping in the Age of Digital Reproduction ", Journal of Southern African Studies, 40(3), pp. 499519 .

Chamelot Fabienne, HiRibarRen Vincent et Rodet Marie (2020), "Archives, the Digital Turn, and Governance in Africa », History in Africa, 47, pp. 101-118.

Daly Samuel Fury Childs (2017), « Archival Research in Africa », African Affairs, 116(463), pp. 311-320.

EluIS Stephen (2002), «Writing Histories of Contemporary Africa », The Journal of African History, 43(1), pp. 1-26.

FOLEY Catherine (2014), « Developing Materials for a Digital Library Gallery », Islamic Africa, 5(1), pp. 8390.

Fouéré Marie-Aude, Rillon Ophélie et POMmerolle Marie-Emmanuelle (2020), «Pourquoi Sources? Rigueur empirique, réflexivité et archivage en sciences humaines et sociales et dans les études africaines », Sources, 1, pp. 1-21.

HANSEN David (2016), « Digitizing Orphan Works: Legal Strategies to Reduce Risks for Open Access to Copyrighted Orphan Works», in K. K. COURTNeY et P. SUBER (dir.), Harvard Library, en ligne. URL : https://dash.harvard.edu/handle/1/27840430 (consulté le 5 décembre 2021).

LIMB Peter (2014), «Islamic Africa: A Select, Annotated Webography », Islamic Africa, 5(1), pp. 91-102.

(2007), " The Politics of Digital "Reform and Revolution": Towards Mainstreaming and African Control of African Digitisation », Innovation, 34, pp. 18-27.

MADORE Frédérick (2020), «Francophone Muslim Intellectuals, Islamic Associational Life and Religious Authority in Burkina Faso », Africa, 90(3), pp. 625-646.

(2016), "The New Vitality of Salafism in Côte d'Ivoire: Toward a Radicalization of Ivoirian Islam? », Journal of Religion in Africa, 46(4), pp. 417-452.

(2018), "Rivalités et collaborations entre aînés et cadets sociaux dans les milieux associatifs islamiques en Côte d'Ivoire et au Burkina Faso (1970-2017) », thèse de doctorat, Québec, Université Laval, en ligne. URL : https://corpus.ulaval.ca/jspui/handle/20.500.11794/33246 (consulté le 5 décembre 2021).

MADORE Frédérick et TRAORÉ Yssoufou (2018), "L'organisation du hadjen Côte d'Ivoire : entre facteur de cohésion et source de rivalités au sein de la communauté musulmane (1993-2010) », Cahiers d'études africaines, 229, pp. 179-208.

MARTINO Enrique (2014), "Open Sourcing the Colonial Archive - A Digital Montage of the History of Fernando Pó and the Bight of Biafra », History in Africa, 41, pp. 387-415.

Marzo Pietro et Gomez-Perez Muriel (2020), «Faire du terrain au Moyen-Orient et en Afrique : stratégies d'approche et défis de positionnement du chercheur », Recherches qualitatives, 39(1), pp. 1-20.

ØSтEBø Terje (2015), «African Salafism: Religious Purity and the Politicization of Purity », Islamic Africa, 6(1-2), pp. 1-29.

PIRON Florence, RÉGULus Samuel et Dibounje Madiba Marie Sophie (dir.) (2016), Justice cognitive, libre accès et savoirs locaux: pour une science ouverte, juste, au service du développement local durable, Québec, Éditions science et bien commun. 
PRUD'HOMme Pierre (2015), "L'imam, l'expert et le diplomate : retour sur une recherche au Haut Conseil Islamique du Mali », Civilisations, 64(1), pp. 129-140.

RoBINSON David (2014), "Muslim Societies in West Africa: Historical and Contemporary Perspectives in Digital Form », Islamic Africa, 5(1), pp. 103-121.

SCHWEDlen Jilian (2006), "The Third Gender: Western Female Researchers in the Middle East », Political Science \& Politics, 39, pp. 425-428.

SHRINGARPURE Bhakti (2020), "Africa and the Digital Savior Complex », Journal of African Cultural Studies, 32(2), pp. 178-194.

Theimer Kate (2012), " Archives in Context and as Context », Journal of Digital Humanities, 1(2), en ligne. URL : $\quad$ http://journalofdigitalhumanities.org/1-2/archives-in-context-and-as-context-by-kate-theimer/ (consulté le 5 décembre 2021).

WHITE Luise (2015), «Introduction - Suitcases, Roads, and Archives: Writing the History of Africa after $1960 »$, History in Africa, 42, pp. 265-267.

White Luise, Miescher Stephan et Cohen David William (dir.) (2001), African Words, African Voices: Critical Practices in Oral History, Bloomington, Indiana University Press. 